\title{
Biogeographical affinities of fish associated to the shrimp trawl fishery in the Gulf of Tehuantepec, Mexico
}

\author{
Marco A. Martínez-Muñoz ${ }^{1}$, Domènec Lloris ${ }^{2}$, Adolfo Gracia ${ }^{1}$, Ricardo Ramírez-Murillo ${ }^{3}$, Saul \\ Sarmiento-Nafáte 4 , Sebastián Ramos-Cruz ${ }^{4} \&$ Felipe Fernández ${ }^{5}$ \\ 1. Unidad Académica de Ecología Marina, Instituto de Ciencias del Mar y Limnología, Universidad Nacional Autónoma \\ de México, PO Box 70-305, México, D.F., 04510; marcoam@unam.mx, gracia@unam.mx \\ 2. Institut de Ciències del Mar (CMIMA-CSIC), Pasage Marítim de la Barceloneta 37-49 Barcelona, Spain; \\ ictios@gmail.com \\ 3. Instituto de Educación Media Superior del Distrito Federal (IEMS-DF). Plantel Tlalpan I. Av. División del Norte \# \\ 906, Col. Narvarte Poniente, 03020, Del. Benito Juárez México, D.F.; hippoglossina@hotmail.com \\ 4. Instituto Nacional de Pesca, Centro Regional de Investigación Pesquera (CRIP) Salina Cruz, Prol. Playa Abierta S/N, \\ Col. Miramar, 70680. Salina Cruz, Oaxaca, México; nafatess@yahoo.com.mx, ramoscruz@yahoo.com \\ 5. Departament d'Ecologia, Facultat de Biologia, Universitat de Barcelona, Diagonal 645, 08028 Barcelona, Spain; \\ ffernandez@ub.edu
}

\section{Received 05-V-2015. Corrected 20-X-2015. Accepted 20-XI-2015.}

\begin{abstract}
Fish by-catch of shrimp fishery from the Gulf of Tehuantepec is composed of several species that are mainly discarded. In this study, fish by-catch species composition, distribution and biogeographical affinities were analyzed. For this, a total of 15 cruises were carried out on the continental shelf, at depths from 15 to $64 \mathrm{~m}$, during 2003, 2004, 2005 and 2013. Results showed that fish by-catch was represented by 58 families, 129 genera and 242 species. The families Haemulidae, Sciaenidae, Paralichthyidae, Gerreidae and Carangidae accounted for $>70 \%$ of the catch. Haemulopsis axillaris, Syacium ovale, Selene peruviana, Diapterus peruvianus, Larimus acclivins and Stellifer erycimba were the most frequent species at $<40 \mathrm{~m}$ depth (inner shelf), and Prionotus stephanophrys, Scorpaena russula, Porichthys analis and Synodus scituliceps were dominant at 40-60 m depth (outer shelf). Analysis of biogeographical affinities showed that $36.1 \%$ of species had a wide distribution, from San Diego Province to the Panamic Province, while $13.2 \%$ had a restricted distribution in the Mexican and Panamic Provinces. The ichthyofaunal composition was markedly influenced by the local environment and seasonal conditions. Rev. Biol. Trop. 64 (2): 683-700. Epub 2016 June 01.
\end{abstract}

Key words: biogeography, ichthyofauna, shrimp, fisheries, by-catch, Gulf of Tehuantepec.

The Gulf of Tehuantepec is influenced by an array of environmental factors like the current pattern, seasonal wind regimes, seasonal upwellings, rivers runoff and extensive coastal lagoon systems, that makes this region a very productive one in terms of fisheries (TapiaGarcía, 1998).

A valuable trawl shrimp fishery is conducted in this area mainly based on four species: Litopenaeus vannamei (White shrimp), Farfantepenaeus californiensis (Yellowleg shrimp), Litopenaeus stylirostris (Blue shrimp) and Farfantepenaeus brevirostris (Crystal shrimp)
(Cervantes-Hernández, Gallardo-Berumen, Ramos-Cruz, Gómez-Ponce \& Gracia, 2008). Gulf of Tehuantepec shrimp catch contributes nearly $5 \%(1880 \mathrm{~m})$ to the total annual catch of the Mexican Pacific (37600 m). Brown and white shrimp are the most important species as they represent $90 \%$ of the total shrimp catch in the area.

Shrimp is the target species in trawling operations, but is associated with a highly diverse fish fauna, which is usually discarded. Both, the large catches discarded and the high ichthyofaunal diversity have caused concern 
and prompted attempts to improve its use and management (Allsop, 1985; Andrew \& Pepperell, 1992; Hendrickson \& Griffin, 1993; Griffiths, Larson, \& Courtney, 2004; Sarmiento-Náfate, Gil-López, \& Arroyo, 2007; Stobutzki, Miller, Jones, \& Salini, 2001).

Studies on the Gulf of Tehuantepec demersal community caught in trawl nets include that of Acal \& Arias (1990), who pointed out the high diversity in fish communities in the region. Bianchi (1991) studied the demersal faunal assemblages (fish, crustaceans and cephalopods) of the shelf and upper slope in a wide area of the Gulf of Tehuantepec. Tapia-García, García-Abad, González-Medina, Macuitl-Montes, and Cerdenares-Ladrón de Guevara (1994) recorded composition and abundance of fish reporting 178 species, with the greatest diversity located in front of the lagoon-estuarine systems of Oaxaca and Chiapas coasts. The dominant species were: Syacium latifrons, S. ovale, Eucinostomus gracilis, Bothus constellatus, Orthopristis chalceus and Pomadasys nitidus. Siqueiros-Beltrones and De la Cruz-Agüero (2004) stood out the importance of taxonomic lists and the analysis of biogeographical distribution as essential tools for fishery management. According to this, several studies have recently assumed increased interest in the Southeastern Mexican Pacific Ocean (Aguilar-Palomino, Mariscal, Gonzalez, \& Rodríguez, 1996; Madrid-Vera, Ruiz, \& Rosado, 1998; Moncayo-Estrada, CastroAguirre, \& De la Cruz-Agüero, 2006). In spite of the ecological and fisheries importance of the Gulf of Tehuantepec, there are only a few by-catch studies on the demersal species from shrimp trawl fishery in this region (Tapia-García, 1998; Tapia-García \& García-Abad, 1998).

Up to now, studies are scarce, spatially and temporally scattered with little comprehensive integration and mainly focused on the fishery perspective. This study presents an updated systematic list of by-catch fishes from shrimp fishery, its abundance and analyzes of their biogeographical relationships.

\section{MATERIALS AND METHODS}

By-catch sampling was carried out during the shrimp closed seasons (April-August) in the Southern Pacific region of Mexico. A total of 15 exploratory cruises were developed during 2003 (five), 2004 (two), 2005 (four) and then resumed in 2013 (four) in a wide section of the continental shelf of the Gulf of Tehuantepec between Punta Chipehua (Oaxaca) and

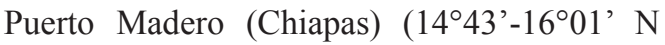
- $\left.92^{\circ} 53^{\prime}-95^{\circ} 22^{\prime} \mathrm{W}\right)$. A sampling design of 62 stations was established for all cruises based on previous studies done in the area (Reyna Cabrera \& Ramos-Cruz, 1998). Total number of locations sometimes varied due to weather conditions; however, this number was never above $25 \%$ (Fig. 1).

Samples were obtained day and night with twin shrimp nets $(24.4 \mathrm{~mm}$ horizontal mouth opening, $40.0 \mathrm{~mm}$ stretched mesh body, 38.1 $\mathrm{mm}$ stretched mesh cod-end) towed at each side of the vessel. The net had a turtle excluder device (TED) fitted in front of the codend. Each trawl had one-hour duration at an average speed of 1.5-2.5 knots; 344 hauls were done in a 15-64 m depth range; 206 hauls $(60 \%)$ were made during the day and $138(40 \%)$ at night; 234 hauls (68\%) were made below $40 \mathrm{~m}$ depth and 110 trawls (32\%) were carried above $40 \mathrm{~m}$ depth.

The catch from each trawl was cast on deck, and total weigth per trawl was estimated filling crates or boxes of $40 \mathrm{~kg}$. A sample of $20 \mathrm{~kg}$ was taken; previous analysis sowed that this was enough to reflect adequately the species composition. Each sample was kept in the freezer at $-30^{\circ} \mathrm{C}$.

The biological material was identified and processed in the laboratories of the Regional Centre of Fisheries Research, Salina Cruz (CRIP-SC). The standard length of each fish was measured with a standard icthyometer of $50 \mathrm{~cm}$ length and a precision of $0.05 \mathrm{~cm}$. Large individual fish were weighted with a scale (25 $\mathrm{g}$ of precision) and small fishes were weighted with an OHAUS digital scale of 2.6 $\mathrm{kg}$ of capacity and a precision of $0.05 \mathrm{~g}$. In the 


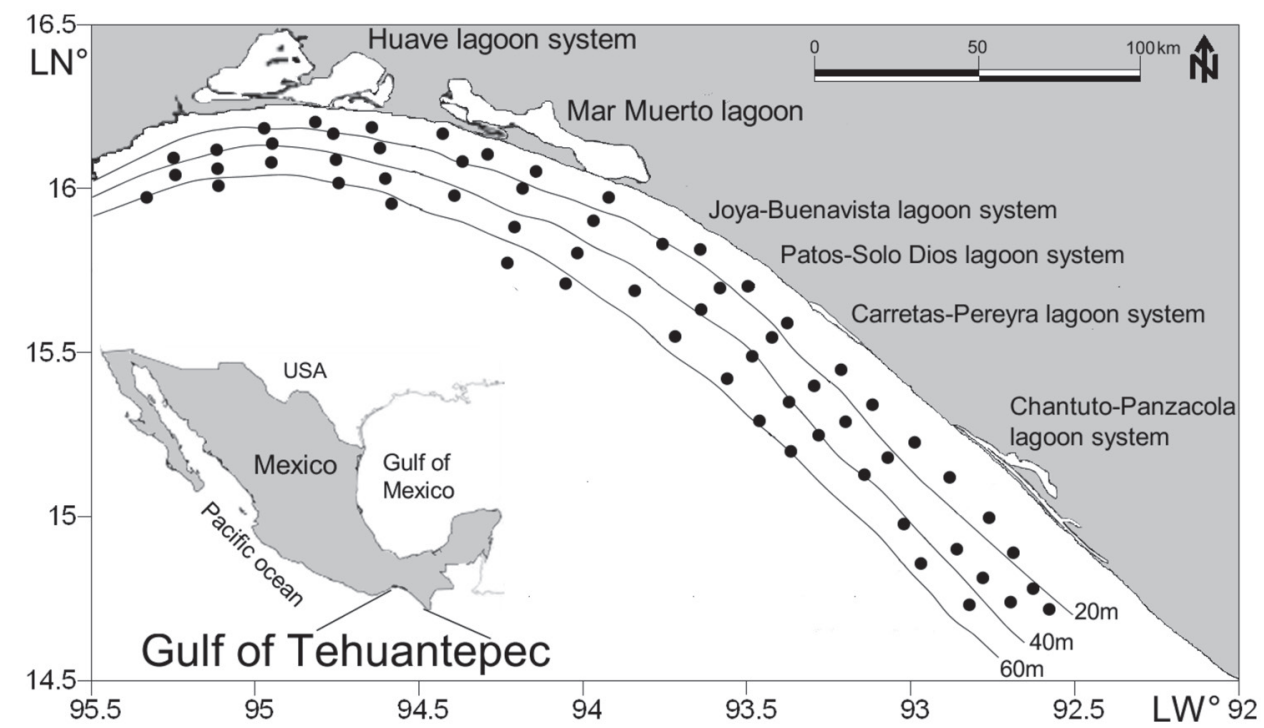

Fig. 1. Study area and sampling stations in the Gulf of Tehuantepec, Mexico.

Fig. 1. Área de estudio y estaciones de muestreo en el Golfo de Tehuantepec, México.

laboratory, fish were separated, washed, relabelled and stored in $70 \%$ isopropyl alcohol.

Fish identification followed the keys of Castro-Aguirre (1978) Eschmeyer (1998), Bussing and López (1993), Fischer, Krupp, Schneider, Sommer, Carpenter and Niem (1995), Robertson and Allen (2002), and AmezcuaLinares (1996). Specialized literature for some specific groups was also consulted: rays, Castro-Aguirre and Espinosa-Pérez (1996); sharks, Espinosa-Pérez, Castro-Aguirre and HuidobroCampos (2004); Sciaenidae, McPhail (1958); Pleuronectiformes, Norman (1934), and Ginsburg (1958); Diplectrum, Rosemblatt and Johnson (1974); and Porichthys, Walter and Rosemblatt (1988).

Systematic ordination followed the criteria of Nelson (2006). Genera and their species are presented in alphabetical order. Common names were taken from Robertson and Allen (2002); Nelson et al. (2004); and Love, Mecklenburg, Mecklenburg and Thorsteinson (2005).

The relative abundance of each species was estimated from the number of individuals within the total of individuals sampled. The species were grouped in four categories: (A) Abundant, relative abundance $>1 \%$; (F)
Frequent, 0.1-0.99 \%; (C) Common, 0.01$0.099 \%$; and (R) Rare, $<0.01 \%$. Differences in biomass values between day and night as well as the depth intervals were calculated through variance analysis (ANOVA) and t-test (Zar, 1999).

Analysis of zoogeographic affinities followed the basic scheme of Briggs (1974, 1995) and Walker (1960), with modifications proposed by Boschi (2000), Galván-Magaña, Gutiérrez-Sánchez, Abitia-Cárdenas and Rodríguez-Romero (2000), Hastings (2000), Robertson \& Allen (2002), Robertson, Grove and McCoster (2004), and Horn, Allen and Lea (2006), considering the following provinces: A) Oregonian: from Puget Sound to Punta Concepción in California. The Southern section is considered a transition zone between this province and that of San Diego. B) San Diego: from Punta Concepción to Bahía Magdalena in the South of Baja California, with temperate-warm waters. C) Cortés: Southern Bahía Magdalena and the Gulf of California. D) Mexican: from Mazatlán, Sinaloa to Tehuantepec (Mexico). E) Panamic: from the South of Salvador to Cabo Blanco (Peru). F) Tropical Eastern Pacific: includes the West coast of the American 
continent between $25^{\circ} \mathrm{N}$ in the Southern part of Bahía Magdalena and $5^{\circ} \mathrm{S}$ at Cabo Blanco, Northern Peru. Differences in species biogeographic affinities between the years 2003 and 2013 were calculated by analysis of variance (ANOVA) (Zar, 1999).

The analysis also considered species with wide biogeographic distribution, such as the Circumtropical, Trans-Pacific and amphiAmerican species of the Central American Isthmus, the Tropical Eastern Pacific and the Western Atlantic.

Classification of the abundant, frequent, common and rare species used the OlmsteadTukey method modified by Sokal and Rohlf
(1995). The data matrices were expressed as frequency of occurrence within each sampling period. Then the frequency of occurrence per cruise was calculated (mean \pm standard deviation). Richness was considered as the number of fish species in the study area.

\section{RESULTS}

The total number of samplings from all cruises was 344, with a total weight of 2818 $\mathrm{kg}$. The 66908 individual fishes represented two Classes, 20 Orders, 58 families, 129 genera and 242 species (Table 1). Previous studies in the area showed a lower number of demersal

TABLE 1

Fishes of the Gulf of Tehuantepec, Mexico

CUADRO 1

Peces del Golfo de Tehuantepec, México

\begin{tabular}{|c|c|c|c|c|}
\hline Species & A & $\mathrm{B}$ & $\mathrm{C}$ & $\mathrm{D}$ \\
\hline \multicolumn{5}{|l|}{ Phylum Chordata } \\
\hline \multicolumn{5}{|l|}{ Class Chondrichthyes } \\
\hline \multicolumn{5}{|l|}{ Subclass Elasmobranchii } \\
\hline \multicolumn{5}{|l|}{ Subdivision Selachii } \\
\hline \multicolumn{5}{|l|}{ Order Carcharhiniformes } \\
\hline \multicolumn{5}{|l|}{ Family Triakidae } \\
\hline Mustelus lunulatus Jordan \& Gilbert, 1883 & Sicklefin smooth-hound & $94-108$ & $\mathrm{R}$ & PS-PP \\
\hline \multicolumn{5}{|l|}{ Family Carcharhinidae } \\
\hline Rhizoprionodon longurio (Jordan \& Gilbert 1882) & Pacific sharpnose shark & 230 & $\mathrm{R}$ & PS-PP \\
\hline \multicolumn{5}{|l|}{ Family Sphyrnidae } \\
\hline Sphyrna lewini (Griffith \& Smith, 1834) & Scalloped hammerhead & $176-350$ & $\mathrm{R}$ & $\mathrm{CT}$ \\
\hline Sphyrna tiburo ( Linnaeus, 1758) & Bonnethead & 260 & $\mathrm{R}$ & PS-PP \\
\hline \multicolumn{5}{|l|}{ Subdivision Batoidea } \\
\hline \multicolumn{5}{|l|}{ Order Torpediniformes } \\
\hline \multicolumn{5}{|l|}{ Family Narcinidae } \\
\hline Narcine entemedor Jordan \& Starks, 1895 & Giant electric ray & $40-399$ & $\mathrm{C}$ & PC-PP \\
\hline Narcine vermiculatus Breder, 1928 & Vermiculate electric ray & $44-444$ & $\mathrm{~F}$ & POT \\
\hline \multicolumn{5}{|l|}{ Order Rajiformes } \\
\hline \multicolumn{5}{|l|}{ Family Rhinobatidae } \\
\hline Rhinobatos glaucostigma Jordan \& Gilbert, 1883 & Speckled guitarfish & $60-335$ & $\mathrm{~F}$ & POT \\
\hline Rhinobatos leucorhynchus Günther, 1867 & Whitesnout guitarfish & $68-571$ & $\mathrm{C}$ & PC-PP \\
\hline Zapteryx exasperata (Jordan \& Gilbert, 1880) & Banded guitarfish & $95-230$ & $\mathrm{C}$ & PS-PP \\
\hline Zapteryx xyster Jordan \& Evermann, 1896 & Witch guitarfish & 406 & $\mathrm{R}$ & PC-PP \\
\hline \multicolumn{5}{|l|}{ Order Myliobatiformes } \\
\hline \multicolumn{5}{|l|}{ Family Urolophidae } \\
\hline Urolophus halleri Cooper, 1863 & Haller's round ray & 170 & $\mathrm{R}$ & POT \\
\hline
\end{tabular}


TABLE 1 (Continued) / CUADRO 1 (Continuación)

\begin{tabular}{|c|c|c|c|c|}
\hline Species & $\mathrm{A}$ & B & $\mathrm{C}$ & $\mathrm{D}$ \\
\hline Urolophus maculatus (Garman, 1913) & Spotted round ray & $110-125$ & $\mathrm{R}$ & PS-PC \\
\hline Urotrygon aspidura (Jordan \& Gilbert, 1882) & Spiny-tail round ray & $41-395$ & $\mathrm{~F}$ & PM-PP \\
\hline Urotrygon chilensis (Günther, 1872) & Chilean round ray & $35-364$ & A & PC-PP \\
\hline Urotrygon munda Gill, 1863 & Munda round ray & $42-230$ & $\mathrm{~F}$ & PM-PP \\
\hline Urotrygon nana Miyake \& McEachran, 1988 & Dwarf round ray & $30-235$ & $\mathrm{~F}$ & PM-PP \\
\hline Urotrygon reticulata Miyake \& McEachran, 1988 & Reticulate round ray & $90-151$ & $\mathrm{R}$ & PM-PP \\
\hline Urotrygon rogersi (Jordan \& Starks, 1895) & Roger's round ray & $64-411$ & $\mathrm{C}$ & PC-PP \\
\hline \multicolumn{5}{|l|}{ Family Dasyatidae } \\
\hline Dasyatis brevis (Garman, 1880) & Whiptail stingray & 146 & $\mathrm{R}$ & PO-PP \\
\hline Dasyatis longa (Garman, 1880) & Longtail stingray & $118-227$ & $\mathrm{R}$ & PO-PP \\
\hline \multicolumn{5}{|l|}{ Family Gymnuridae } \\
\hline Gymnura marmorata (Cooper,1864) & California butterfly ray & $200-326$ & $\mathrm{C}$ & PS-PP \\
\hline \multicolumn{5}{|l|}{ Family Myliobatidae } \\
\hline Aetobatus narinari (Euphrasen, 1790) & Spotted eagle ray & $165-195$ & $\mathrm{C}$ & $\mathrm{CT}$ \\
\hline Rhinoptera steindachneri Evermann \& Jenkins, 1891 & Pacific cownose ray & $175-406$ & $\mathrm{R}$ & PS-PP \\
\hline \multicolumn{5}{|l|}{ Class Actinopterygii } \\
\hline \multicolumn{5}{|l|}{ Subclass Neopterygii } \\
\hline \multicolumn{5}{|l|}{ Division Teleostei } \\
\hline \multicolumn{5}{|l|}{ Order Albuliformes } \\
\hline \multicolumn{5}{|l|}{ Family Albulidae } \\
\hline Albula nemoptera (Fowler, 1911) & Threadfin bonefish & $134-276$ & $\mathrm{C}$ & POT \\
\hline Albula vulpes (Linnaeus, 1758) & Bonefish & $175-231$ & $\mathrm{C}$ & POT \\
\hline \multicolumn{5}{|l|}{ Order Anguilliformes } \\
\hline \multicolumn{5}{|l|}{ Suborder Muraenoidei } \\
\hline \multicolumn{5}{|l|}{ Family Muraenidae } \\
\hline Gymnothorax equatorialis (Hildenbrand,1946) & Spotted-tail moray & $213-681$ & $\mathrm{C}$ & PC-PP \\
\hline Gymnothorax panamensis (Steindachner, 1876) & Panamic moray & $531-569$ & $\mathrm{C}$ & PS-PP \\
\hline \multicolumn{5}{|l|}{ Suborder Congroidei } \\
\hline \multicolumn{5}{|l|}{ Family Ophichthidae } \\
\hline Myrophis vafer Jordan \& Gilbert, 1883 & Pacific worm eel & 247 & $\mathrm{R}$ & PS-PP \\
\hline Ophichthus zophochir Jordan \& Gilbert, 1882 & Yellow snake eel & $205-580$ & $\mathrm{C}$ & PS-PP \\
\hline Pseudomyrophis micropinna Wade, 1946 & Smallfin worm eel & 169 & $\mathrm{R}$ & PM-PP \\
\hline \multicolumn{5}{|l|}{ Family Congridae } \\
\hline Ariosoma gilberti (Ogilby, 1898) & Gilbert's garden eel & $109-165$ & $\mathrm{C}$ & $\mathrm{PC}$ \\
\hline Paraconger californiensis Kanazawa, 1961 & California conger & 483 & $\mathrm{R}$ & PS-PP \\
\hline \multicolumn{5}{|l|}{ Order Clupeiformes } \\
\hline \multicolumn{5}{|l|}{ Suborder Clupeoidei } \\
\hline \multicolumn{5}{|l|}{ Family Engraulidae } \\
\hline Anchoa argentivittata (Regan, 1904) & Regan's anchovy & $50-65$ & $\mathrm{R}$ & PC-PP \\
\hline Anchoa helleri (Hubbs, 1921) & Heller's anchovy & $38-109$ & $\mathrm{C}$ & PC-PM \\
\hline Anchoa ischana ( Jordan \& Gilbert, 1882) & Slender anchovy & $40-119$ & $\mathrm{~F}$ & PC-PP \\
\hline Anchoa lucida (Jordan \& Gilbert, 1882) & Bright anchovy & $46-193$ & $\mathrm{~F}$ & PC-PP \\
\hline Anchoa mundeola (Gilbert \& Pierson, 1898) & False Panama anchovy & $50-72$ & $\mathrm{C}$ & PC-PP \\
\hline Anchoa nasus (Kner \& Steindachner, 1867) & Longnose anchovy & $60-119$ & $\mathrm{C}$ & PC-PP \\
\hline Anchoa starksi (Gilbert \& Pierson, 1898) & Black-tail anchovy & 84 & $\mathrm{R}$ & PP \\
\hline Anchoa walkeri Baldwin \& Chang, 1970 & Walker's anchovy & $40-145$ & $\mathrm{C}$ & PC-PP \\
\hline Anchovia macrolepidota (Kner, 1863) & Bigscale anchovy & $47-177$ & $\mathrm{~F}$ & PC-PM \\
\hline
\end{tabular}


TABLE 1 (Continued) / CUADRO 1 (Continuación)

\begin{tabular}{|c|c|c|c|c|}
\hline Species & $\mathrm{A}$ & $\mathrm{B}$ & $\mathrm{C}$ & $\mathrm{D}$ \\
\hline Cetengraulis mysticetus (Günther, 1867) & Pacific anchoveta & $36-134$ & $\mathrm{C}$ & PS-PP \\
\hline \multicolumn{5}{|l|}{ Family Clupeidae } \\
\hline Harengula thrissina Jordan \& Gilbert, 1882 & Pacific flatiron herring & $117-180$ & $\mathrm{C}$ & PC-PP \\
\hline Lile gracilis Castro-Aguirre \& Vivero, 1990 & Graceful piquitinga & $80-190$ & $\mathrm{C}$ & PC-PP \\
\hline Lile stolifera (Jordan \& Gilbert, 1882) & Pacific piquitinga & 118 & $\mathrm{R}$ & PC-PP \\
\hline Opisthonema libertate ( Günther, 1867) & Pacific thread herring & $73-195$ & $\mathrm{~F}$ & PS-PP \\
\hline Opisthonema medirastre Berry \& Barrett, 1963 & Middling thread herring & $118-166$ & $\mathrm{C}$ & PC-PP \\
\hline Sardinops sagax (Jenyns, 1842) & South American pilchard & 207 & $\mathrm{R}$ & PA-PC \\
\hline \multicolumn{5}{|l|}{ Family Pristigasteridae } \\
\hline Opisthopterus dovii ( Günther, 1868) & Dove's longfin herring & $59-180$ & $\mathrm{~F}$ & PS-PP \\
\hline Pliosteostoma lutipinnis ( Jordan \& Gilbert, 1882) & Yellowfin herring & $45-482$ & $\mathrm{~F}$ & PM-PP \\
\hline \multicolumn{5}{|l|}{ Order Siluriformes } \\
\hline \multicolumn{5}{|l|}{ Family Ariidae } \\
\hline Ariopsis guatemalensis (Günther, 1864) & Blue sea catfish & $66-195$ & $\mathrm{~F}$ & PC-PP \\
\hline Ariopsis seemanni (Günther, 1864) & Tete sea catfish & $41-236$ & F & PC-PP \\
\hline Bagre panamensis (Gill, 1863) & Chilhuil sea catfish & $64-410$ & $\mathrm{~F}$ & PC-PP \\
\hline Bagre pinnimaculatus (Steindachner, 1876) & Red sea catfish & $152-325$ & $\mathrm{R}$ & PC-PP \\
\hline Cathorops dasycephalus (Günther, 1864) & Big-bellied sea catfish & $38-172$ & $\mathrm{C}$ & PM \\
\hline Cathorops fuerthii (Steindachner, 1876) & Congo sea catfish & $40-205$ & $\mathrm{~F}$ & PC-PP \\
\hline Cathorops steindachneri (Gilbert \& Starks,1904) & Steindachner's sea catfish & $119-191$ & $\mathrm{C}$ & PM-PP \\
\hline Cathorops taylori (Hildebrand, 1925) & Sea catfish & $176-213$ & $\mathrm{R}$ & PP \\
\hline Galeichthys peruvianus Lütken, 1874 & Peruvian sea catfish & $140-241$ & $\mathrm{C}$ & PM-PP \\
\hline Notarius kessleri (Steindachner, 1876) & Sculptured sea catfish & $70-255$ & $\mathrm{C}$ & PM-PP \\
\hline Notarius planiceps (Steindachner, 1876) & Flathead sea catfish & $68-247$ & $\mathrm{C}$ & PM-PP \\
\hline Notarius troschelii (Gill, 1863) & Chili sea catfish & $28-275$ & $\mathrm{~F}$ & PM-PP \\
\hline Occidentarius platypogon (Günther, 1864) & Cominate sea catfish & $57-290$ & $\mathrm{~F}$ & PC-PP \\
\hline Sciades dowii (Gill, 1863) & Brown sea catfish & $170-190$ & $\mathrm{R}$ & PP \\
\hline \multicolumn{5}{|l|}{ Order Aulopiformes } \\
\hline \multicolumn{5}{|l|}{ Suborder Synodontoidei } \\
\hline \multicolumn{5}{|l|}{ Family Synodontidae } \\
\hline Synodus evermanni Jordan \& Bollman, 1890 & Inotted lizardfish & $84-312$ & F & PS-PP \\
\hline Synodus scituliceps Jordan \& Gilbert, 1882 & Shorthead lizardfish & $91-420$ & $\mathrm{~F}$ & PS-PP \\
\hline \multicolumn{5}{|l|}{ Order Gadiformes } \\
\hline \multicolumn{5}{|l|}{ Family Merluccidae } \\
\hline Merluccius angustimanus Garman, 1899 & Panama hake & $68-155$ & $\mathrm{C}$ & PS-PP \\
\hline Merluccius productus (Ayres, 1855) & North Pacific hake & $46-180$ & $\mathrm{C}$ & PS-PP \\
\hline \multicolumn{5}{|l|}{ Order Ophidiiformes } \\
\hline \multicolumn{5}{|l|}{ Suborder Ophidioidei } \\
\hline \multicolumn{5}{|l|}{ Family Ophidiidae } \\
\hline Brotula clarkae Hubbs, 1944 & Pacific bearded brotula & $154-315$ & $\mathrm{C}$ & PC-PP \\
\hline Chilara taylori (Girard,1858) & Spotted cusk-eel & 178 & $\mathrm{R}$ & PO-PP \\
\hline Lepophidium pardale (Gilbert, 1890) & Leopard cusk eel & $120-169$ & $\mathrm{R}$ & POT \\
\hline Lepophidium prorates (Jordan \& Bollman, 1890) & Prowspine cusk eel & $60-254$ & $\mathrm{C}$ & POT \\
\hline Ophidion galeoides (Gilbert, 1890) & Striped cusk eel & 175 & $\mathrm{R}$ & PM-PP \\
\hline Otophidium indefatigabile Jordan \& Bollman, 1890 & Bighead cusk eel & 169 & $\mathrm{R}$ & PS-PP \\
\hline \multicolumn{5}{|l|}{ Order Batrachoidiformes } \\
\hline \multicolumn{5}{|l|}{ Family Batrachoididae } \\
\hline Batrachoides boulengeri Gilbert \& Starks, 1904 & Boulenger's toadfish & $87-210$ & $\mathrm{C}$ & PM-PP \\
\hline
\end{tabular}


TABLE 1 (Continued) / CUADRO 1 (Continuación)

\begin{tabular}{|c|c|c|c|c|}
\hline Species & $\mathrm{A}$ & B & $\mathrm{C}$ & $\mathrm{D}$ \\
\hline Batrachoides waltersi Collette \& Russo, 1981 & Walter's toadfish & $40-292$ & $\mathrm{~F}$ & PM-PP \\
\hline Porichthys analis Hubbs \& Schultz, 1939 & Darkedge midshipman & $10-174$ & A & PS-PP \\
\hline Porichthys margaritatus (Richardson, 1844) & Daisy midshipman & $52-160$ & $\mathrm{~F}$ & POT \\
\hline \multicolumn{5}{|l|}{ Suborder Antennariidae } \\
\hline \multicolumn{5}{|l|}{ Family Antennariidae } \\
\hline Fowlerichthys avalonis (Jordan \& Starks, 1907) & Roughbar frogfish & $50-156$ & $\mathrm{C}$ & PS-PP \\
\hline \multicolumn{5}{|l|}{ Order Mugiliformes } \\
\hline \multicolumn{5}{|l|}{ Family Mugilidae } \\
\hline Mugil cephalus Linnaeus, 1758 & Flathead grey mullet & $167-188$ & $\mathrm{R}$ & PS-PP \\
\hline Mugil curema Valenciennens, 1836 & White mullet & $160-210$ & $\mathrm{C}$ & PC-PP \\
\hline Mugil setosus Gilbert, 1892 & Liseta mullet & 155 & $\mathrm{R}$ & PC-PP \\
\hline \multicolumn{5}{|l|}{ Suborder Belonoidei } \\
\hline \multicolumn{5}{|l|}{ Family Exocoetidae } \\
\hline Cheilopogon papilio (Clark,1936) & Butterfly flyingfish & $117-127$ & $\mathrm{R}$ & PM-PP \\
\hline \multicolumn{5}{|l|}{ Order Beloniformes } \\
\hline \multicolumn{5}{|l|}{ Family Hemiramphidae } \\
\hline Cypselurus callopterus (Günther, 1866) & Ornamented flyingfish & 185 & $\mathrm{R}$ & PS-PP \\
\hline Hemiramphus saltator Gilbert \& Starks, 1904 & Longfin halfbeak & $240-250$ & $\mathrm{R}$ & PS-PP \\
\hline \multicolumn{5}{|l|}{ Suborder Cyprinodontoidei } \\
\hline \multicolumn{5}{|l|}{ Order Cyprinodontiformes } \\
\hline \multicolumn{5}{|l|}{ Family Poeciliidae } \\
\hline Poecilia butleri Jordan, 1889 & Pacific molly & 55 & $\mathrm{R}$ & PM \\
\hline Poeciliopsis fasciata (Meek, 1904) & San Jeronimo livebearer & 52 & $\mathrm{R}$ & PS-PP \\
\hline \multicolumn{5}{|l|}{ Order Gasterosteifomes } \\
\hline \multicolumn{5}{|l|}{ Suborder Sygnathoidei } \\
\hline \multicolumn{5}{|l|}{ Family Syngnathidae } \\
\hline Hippocampus ingens Girard, 1858 & Pacific seahorse & $117-197$ & $\mathrm{R}$ & PO-PP \\
\hline \multicolumn{5}{|l|}{ Family Fistularidae } \\
\hline Fistularia commersonii Rüppell, 1838 & Bluespotted cornetfish & 170 & $\mathrm{R}$ & PC-PP \\
\hline Fistularia corneta Gilbert \& Starks, 1904 & Pacific cornetfish & $164-381$ & $\mathrm{C}$ & PO-PP \\
\hline \multicolumn{5}{|l|}{ Order Scorpaeniformes } \\
\hline \multicolumn{5}{|l|}{ Suborder Scorpaenoidei } \\
\hline \multicolumn{5}{|l|}{ Family Scorpaenidae } \\
\hline Pontinus sierra (Gilbert, 1890) & Speckled scorpionfish & 67 & $\mathrm{R}$ & PC-PM \\
\hline Scorpaena histrio Jenyns, 1840 & Player scorpionfish & $35-88$ & $\mathrm{~F}$ & PS-PP \\
\hline Scorpaena mystes Jordan \& Starks, 1895 & Pacific spotted scorpionfish & $92-145$ & $\mathrm{R}$ & PS-PP \\
\hline Scorpaena russula Jordan \& Bollman, 1890 & Reddish scorpionfish & $27-130$ & A & PS-PP \\
\hline \multicolumn{5}{|l|}{ Suborder Platycephaloidei } \\
\hline \multicolumn{5}{|l|}{ Family Triglidae } \\
\hline Bellator gymnostethus (Gilbert, 1892) & Naked-belly searobin & $35-239$ & $\mathrm{R}$ & PC-PP \\
\hline Bellator loxias (Jordan, 1897) & Barred searobin & $65-277$ & $\mathrm{C}$ & POT \\
\hline Bellator xenisma (Jordan \& Bollman, 1889) & Splitnose searobin & $32-282$ & $\mathrm{~F}$ & PS-PP \\
\hline Prionotus albirostris Jordan \& Bollman, 1890 & Whitesnout searobin & $39-110$ & $\mathrm{C}$ & PC-PP \\
\hline Prionotus birostratus Richardson, 1844 & Two-beaked searobin & $55-198$ & $\mathrm{C}$ & PC-PP \\
\hline Prionotus horrens Richardson, 1844 & Bristly searobin & $30-125$ & $\mathrm{~F}$ & POT \\
\hline Prionotus ruscarius Gilbert \& Starks, 1904 & Common searobin & $53-245$ & $\mathrm{~F}$ & PS-PP \\
\hline Prionotus stephanophrys Lockington, 1881 & Lumptail searobin & $30-200$ & A & PM-PP \\
\hline
\end{tabular}


TABLE 1 (Continued) / CUADRO 1 (Continuación)

\begin{tabular}{|c|c|c|c|c|}
\hline Species & A & B & $\mathrm{C}$ & $\mathrm{D}$ \\
\hline \multicolumn{5}{|l|}{ Order Perciformes } \\
\hline \multicolumn{5}{|l|}{ Suborder Percodei } \\
\hline \multicolumn{5}{|l|}{ Family Centropomidae } \\
\hline Centropomus armatus Gill, 1863 & Armed snook & 170 & $\mathrm{R}$ & PM-PP \\
\hline Centropomus medius Günther, 1864 & Blackfin snook & $93-140$ & $\mathrm{C}$ & PS-PP \\
\hline Centropomus robalito Jordan \& Gilbert, 1882 & Yellowfin snook & $58-190$ & $\mathrm{~F}$ & PC-PP \\
\hline Centropomus unionensis Bocourt, 1868 & Union snook & $69-162$ & $\mathrm{C}$ & PM-PP \\
\hline \multicolumn{5}{|l|}{ Family Serranidae } \\
\hline Alphestes immaculatus Breder, 1936 & Pacific mutton hamlet & $65-110$ & $\mathrm{C}$ & PC-PP \\
\hline Alphestes multiguttatus (Günther, 1867) & Rivulated mutton hamlet & $72-172$ & $\mathrm{C}$ & PC-PP \\
\hline Cephalopholis panamensis (Steindachner, 1876) & Pacific graysby & 180 & $\mathrm{R}$ & PC-PP \\
\hline Diplectrum eumelum Rosemblatt \&Johnson, 1974 & Orange-spotted sand perch & $50-162$ & $\mathrm{~F}$ & POT \\
\hline Diplectrum euryplectrum Jordan \& Bollman, 1890 & Bighead sand perch & $59-155$ & F & PC-PP \\
\hline Diplectrum labarum Rosemblatt \& Johnson, 1974 & Highfin sand perch & $60-158$ & $\mathrm{~F}$ & PS-PP \\
\hline Diplectrum macropoma (Günther, 1864) & Mexican sand perch & $65-140$ & $\mathrm{C}$ & POT \\
\hline Diplectrum pacificum Meek \& Hildebrand, 1925 & Inshore sand perch & $43-195$ & F & PS-PP \\
\hline Epinephelus analogus Gill, 1863 & Spotted grouper & $90-308$ & $\mathrm{C}$ & PS-PP \\
\hline Epinephelus labriformis (Jenyns, 1840) & Starry grouper & 104-104 & $\mathrm{R}$ & PC-PP \\
\hline Hyporthodus acanthistius (Gilbert, 1892) & Rooster hind & $74-510$ & $\mathrm{C}$ & PS-PP \\
\hline Hyporthodus exsul (Fowler, 1944) & Tenspine grouper & $200-570$ & $\mathrm{C}$ & PC-PP \\
\hline Hyporthodus niphobles (Gilbert \& Starks, 1897) & Star-studded grouper & $61-138$ & $\mathrm{C}$ & PS-PP \\
\hline Rypticus nigripinnis Gill, 1861 & Blackfin soapfish & $98-160$ & $\mathrm{C}$ & PS-PP \\
\hline \multicolumn{5}{|l|}{ Family Priacanthidae } \\
\hline Pristigenys serrula (Gilbert, 1891) & Popeye catalufa & $45-128$ & $\mathrm{~F}$ & PO-PP \\
\hline \multicolumn{5}{|l|}{ Family Carangidae } \\
\hline Carangoides otrynter (Jordan \& Gilbert, 1883) & Threadfin jack & $36-468$ & $\mathrm{C}$ & PS-PP \\
\hline Caranx caballus Günther, 1868 & Green jack & $132-190$ & $\mathrm{R}$ & PS-PP \\
\hline Caranx vinctus Jordan \& Gilbert, 1882 & Cocinero & $32-175$ & F & PS-PP \\
\hline Caranx caninus Günther, 1867 & Pacific crevalle jack & $91-118$ & $\mathrm{R}$ & PS-PP \\
\hline Chloroscombrus orqueta Jordan \& Gilbert, 1883 & Pacific bumper & $48-325$ & A & PS-PP \\
\hline Decapterus macarellus (Cuvier, 1833) & Mackerel scad & $165-184$ & $\mathrm{R}$ & CT \\
\hline Decapterus macrosoma Bleeker, 1851 & Shortfin scad & $92-192$ & $\mathrm{C}$ & $\mathrm{CT}$ \\
\hline Decapterus muroadsi (Temminck \& Schlengel, 1844) & Amberstripe scad & $115-325$ & $\mathrm{R}$ & $\mathrm{CT}$ \\
\hline Hemicaranx leucurus (Günther, 1864) & Yellowfin jack & $56-190$ & $\mathrm{C}$ & POT \\
\hline Hemicaranx zelotes Gilbert, 1898 & Blackfin jack & $39-195$ & $\mathrm{~F}$ & PC-PP \\
\hline Oligoplites refulgens Gilbert \& Starks, 1904 & Shortjaw leatherjack & 113 & $\mathrm{R}$ & PM-PP \\
\hline Oligoplites saurus (Bloch \& Schneider, 1801) & Leatherjack & 144 & $\mathrm{R}$ & PC-PP \\
\hline Selar crumenophthalmus (Bloch, 1793) & Bigeye scad & $54-195$ & $\mathrm{C}$ & PC-PP \\
\hline Selene brevoortii (Gill, 1863) & Mexican lookdown & $40-205$ & $\mathrm{~F}$ & PC-PP \\
\hline Selene orstedii Lutken, 1880 & Mexican moonfish & $42-78$ & $\mathrm{C}$ & PC-PP \\
\hline Selene peruviana (Guichenot, 1866) & Pacific moonfish & $31-238$ & A & PS-PP \\
\hline \multicolumn{5}{|l|}{ Family Lutjanidae } \\
\hline Lutjanus argentiventris (Peters, 1869) & Yellow snapper & 70 & $\mathrm{R}$ & PS-PP \\
\hline Lutjanus guttatus (Steindachner, 1869) & Spotted rose snapper & $42-405$ & $\mathrm{~F}$ & PC-PP \\
\hline Lutjanus peru (Nichols \& Murphy, 1922) & Pacific red snapper & $39-295$ & $\mathrm{~F}$ & PC-PP \\
\hline \multicolumn{5}{|l|}{ Family Gerreidae } \\
\hline Diapterus aureolus Jordan \& Gilbert 1882 & Golden mojarra & $52-157$ & $\mathrm{~F}$ & PC-PP \\
\hline
\end{tabular}


TABLE 1 (Continued) / CUADRO 1 (Continuación)

\begin{tabular}{|c|c|c|c|c|}
\hline Species & A & B & $\mathrm{C}$ & $\mathrm{D}$ \\
\hline Diapterus peruvianus (Cuvier, 1830) & Peruvian mojarra & $10-222$ & A & PC-PP \\
\hline Eucinostomus argenteus Baird \& Girard, 1855 & Silver mojarra & 78-109 & $\mathrm{R}$ & PS-PP \\
\hline Eucinostomus currani Zahuranec, 1980 & Pacific flagfin mojarra & $51-194$ & A & PC-PP \\
\hline Eucinostomus dowii $(\mathrm{Gill}, 1863)$ & Dow's mojarra & $80-145$ & $\mathrm{R}$ & PC-PP \\
\hline Eucinostomus entomelas Zahuranec, 1980 & Dark-spot mojarra & $72-145$ & $\mathrm{C}$ & PC-PP \\
\hline Eucinostomus gracilis (Gill, 1862) & Graceful mojarra & $48-170$ & A & PC-PP \\
\hline Gerres cinereus (Walbaum,1792) & Yellow fin mojarra & $72-92$ & $\mathrm{R}$ & PC-PP \\
\hline \multicolumn{5}{|l|}{ Family Haemulidae } \\
\hline Anisotremus interruptus (Gill, 1862) & Burrito grunt & 132 & $\mathrm{R}$ & PC-PP \\
\hline Conodon serrifer Jordan \& Gilbert, 1882 & Armed grunt & $64-165$ & $\mathrm{~F}$ & PS-PP \\
\hline Haemulon scudderii Gill, 1862 & Grey grunt & $42-200$ & A & PC-PP \\
\hline Haemulon steindachneri (Jordan \& Gilbert, 1882) & Chere-chere grunt & $82-205$ & $\mathrm{C}$ & POT \\
\hline Haemulopsis axillaris (Steindachner, 1869) & Yellowstripe grunt & $31-297$ & A & PC-PP \\
\hline Haemulopsis elongatus (Steindachner, 1879) & Elongate grunt & 132 & $\mathrm{R}$ & PM-PP \\
\hline Haemulopsis leuciscus (Günther, 1864) & White grunt & $31-252$ & $\mathrm{~F}$ & PS-PP \\
\hline Haemulopsis nitidus (Steindachner, 1869) & Shining grunt & $47-285$ & $\mathrm{~F}$ & PC-PP \\
\hline Microlepidotus brevipinnis (Steindachner, 1869) & Humpback grunt & 115 & $\mathrm{R}$ & PC-PP \\
\hline Orthopristis chalceus (Günther, 1864) & Brassy grunt & $72-215$ & $\mathrm{~F}$ & PM \\
\hline Orthopristis reddingi Jordan \& Richardson, 1895 & Bronze-striped grunt & $98-148$ & $\mathrm{C}$ & PC-PP \\
\hline Pomadasys bayanus Jordan \& Evermann, 1898 & Purplemouth grunt & $55-176$ & $\mathrm{C}$ & PC-PP \\
\hline Pomadasys branickii (Steindachner, 1879) & Sand grunt & 111-182 & $\mathrm{C}$ & PC-PP \\
\hline Pomadasys panamensis (Steindachner, 1876) & Panama grunt & $32-314$ & $\mathrm{~F}$ & PC-PP \\
\hline Xenichthys xanti Gill, 1863 & Longfin salema & $64-176$ & $\mathrm{~F}$ & PS-PP \\
\hline Xenistius californiensis (Steindachner, 1876) & Californian salema & $102-130$ & $\mathrm{R}$ & PS-PP \\
\hline \multicolumn{5}{|l|}{ Family Polynemidae } \\
\hline Polydactylus approximans (Lay \& Bennet, 1839) & Blue bobo & $56-207$ & A & PS-PP \\
\hline Polydactylus opercularis (Gill, 1863) & Yellow bobo & $70-260$ & $\mathrm{~F}$ & PC-PP \\
\hline \multicolumn{5}{|l|}{ Family Sciaenidae } \\
\hline Aplodinotus grunniens Rafinesque, 1819 & Freshwater drum & 95-199 & $\mathrm{C}$ & PM \\
\hline Bairdiella armata Gill,1863 & Armed croaker & $68-185$ & $\mathrm{C}$ & PC-PP \\
\hline Bairdiella ensifera (Jordan \& Gilbert, 1862) & Swordspine croaker & $130-156$ & $\mathrm{C}$ & PM-PP \\
\hline Cynoscion phoxocephalus Jordan \& Gilbert, 1882 & Cachema weakfish & 108 & $\mathrm{R}$ & PC-PP \\
\hline Cynoscion reticulatus (Günther, 1864) & Striped weakfish & $160-205$ & $\mathrm{R}$ & PC-PP \\
\hline Cynoscion stolzmanni (Steindachner, 1879) & Stolzmann's weakfish & $83-184$ & $\mathrm{R}$ & PC-PP \\
\hline Elattarchus archidium (Jordan \& Gilbert 1882) & Bluestreak drum & $110-155$ & $\mathrm{C}$ & PC-PP \\
\hline Isopisthus remifer Jordan \& Gilbert, 1882 & Silver weakfish & $50-215$ & $\mathrm{~F}$ & PS-PP \\
\hline Larimus acclivis Jordan \& Bristol, 1898 & Steeplined drum & $34-211$ & A & PC-PP \\
\hline Larimus argenteus (Gill, 1863) & Silver drum & $37-195$ & A & PC-PP \\
\hline Larimus effulgens Gilbert, 1898 & Shining drum & $68-170$ & $\mathrm{~F}$ & PC-PP \\
\hline Larimus pacificus Jordan \& Bollman, 1890 & Pacific drum & $39-155$ & $\mathrm{~F}$ & POT \\
\hline Menticirrhus elongatus (Günther, 1864) & Pacific kingcroaker & $85-280$ & $\mathrm{C}$ & PC-PP \\
\hline Menticirrhus nasus (Günther, 1868) & Highfin kingcroaker & $74-215$ & $\mathrm{~F}$ & PC-PP \\
\hline Menticirrhus panamensis (Steindachner, 1876) & Panama kingcroaker & $54-192$ & $\mathrm{C}$ & PC-PP \\
\hline Menticirrhus undulatus (Girard,1854) & California kingcroaker & $87-185$ & $\mathrm{C}$ & PC-PP \\
\hline Micropogonias altipinnis (Günther, 1864) & Tallfin croaker & $59-280$ & $\mathrm{~F}$ & PC-PP \\
\hline Micropogonias ectenes (Jordan \& Gilbert 1882) & Slender croaker & $189-190$ & $\mathrm{R}$ & PS-PM \\
\hline Nebris occidentalis Vaillant, 1897 & Pacific smalleye croaker & $54-240$ & $\mathrm{~F}$ & PM-PP \\
\hline
\end{tabular}


TABLE 1 (Continued) / CUADRO 1 (Continuación)

\begin{tabular}{|c|c|c|c|c|}
\hline Species & A & $\mathrm{B}$ & $\mathrm{C}$ & $\mathrm{D}$ \\
\hline Ophioscion imiceps (Jordan \& Gilbert 1882) & Blinkard croaker & $50-194$ & $\mathrm{~F}$ & PC-PP \\
\hline Ophioscion strabo Gilbert, 1897 & Squint-eyed croaker & $69-178$ & $\mathrm{C}$ & PM-PP \\
\hline Ophioscion typicus Gill, 1863 & Point-nosed croaker & $70-475$ & $\mathrm{C}$ & PS-PP \\
\hline Paralonchurus goodei Gilbert, 1898 & Goode croaker & $100-182$ & $\mathrm{C}$ & PM-PP \\
\hline Paralonchurus rathbuni (Jordan \& Bollman 1890) & Bearded banded croaker & $112-201$ & $\mathrm{C}$ & PM-PP \\
\hline Stellifer chrysoleuca (Günther, 1867) & Shortnose stardrum & $44-246$ & $\mathrm{C}$ & PS-PM \\
\hline Stellifer ericymba (Jordan \& Gilbert, 1882) & Hollow stardrum & $40-300$ & A & PS-PM \\
\hline Stellifer fuerthii (Steindachner, 1876) & White stardrum & $54-186$ & $\mathrm{~F}$ & PP \\
\hline Stellifer illecebrosus Gilbert, 1898 & Silver stardrum & $59-185$ & $\mathrm{~F}$ & PS-PP \\
\hline Umbrina xanti Gill, 1862 & Polla drum & $45-313$ & $\mathrm{~F}$ & PM-PP \\
\hline \multicolumn{5}{|l|}{ Family Mullidae } \\
\hline Pseudupeneus grandisquamis (Gill, 1863) & Bigscale goatfish & $56-172$ & $\mathrm{~A}$ & PS-PP \\
\hline \multicolumn{5}{|l|}{ Family Chaetodontidae } \\
\hline Chaetodon humeralis Günther, 1860 & Threebanded butterflyfish & $37-112$ & $\mathrm{~F}$ & PS-PP \\
\hline \multicolumn{5}{|l|}{ Suborder Labroidei } \\
\hline \multicolumn{5}{|l|}{ Family Labridae } \\
\hline Halichoeres chierchiae Di Caporiacco, 1948 & Wounded wrasse & $128-152$ & $\mathrm{R}$ & PC-PP \\
\hline \multicolumn{5}{|l|}{ Suborder Trachinoidei } \\
\hline \multicolumn{5}{|l|}{ Family Ephippidae } \\
\hline Chaetodipterus zonatus (Girard, 1858) & Pacific sleeper & $125-140$ & $\mathrm{R}$ & PC-PP \\
\hline Parapsettus panamensis (Steindachner, 1876) & Pennant goby & $59-90$ & $\mathrm{C}$ & PC-PM \\
\hline \multicolumn{5}{|l|}{ Family Uranoscopidae } \\
\hline Astroscopus zephyreus Gilbert \& Starks 1897 & Pacific stargazer & 193 & $\mathrm{R}$ & PS-PP \\
\hline \multicolumn{5}{|l|}{ Suborder Gobioidei } \\
\hline \multicolumn{5}{|l|}{ Family Eleotridae } \\
\hline Gobiomorus maculatus (Günther, 1859) & Tailspot goby & $66-72$ & $\mathrm{R}$ & PC-PP \\
\hline \multicolumn{5}{|l|}{ Family Gobiidae } \\
\hline Bollmannia ocellata Gilbert, 1892 & Pacific spadefish & $32-176$ & $\mathrm{~F}$ & PC-PP \\
\hline Bollmannia stigmatura Gilbert, 1892 & Panama spadefish & $33-286$ & $\mathrm{~F}$ & POT \\
\hline \multicolumn{5}{|l|}{ Suborder Scombroidei } \\
\hline \multicolumn{5}{|l|}{ Family Trichiuridae } \\
\hline Lepidopus fitchi Rosenblatt \& Wilson, 1987 & Pacific scabbardfish & 400 & $\mathrm{R}$ & PO-PP \\
\hline Trichiurus lepturus Linnaeus, 1758 & Largehead hairtail & 436 & $\mathrm{C}$ & $\mathrm{CT}$ \\
\hline \multicolumn{5}{|l|}{ Family Scombridae } \\
\hline Scomberomorus sierra Jordan \& Starks, 1895 & Pacific sierra & $135-290$ & $\mathrm{C}$ & PS-PP \\
\hline \multicolumn{5}{|l|}{ Family Sphyraenidae } \\
\hline Sphyraena ensis Jordan \& Gilbert, 1882 & Mexican barracuda & $16-304$ & $\mathrm{~F}$ & PC-PP \\
\hline \multicolumn{5}{|l|}{ Suborder Stromateoidei } \\
\hline \multicolumn{5}{|l|}{ Family Stromateidae } \\
\hline Peprilus medius (Peters, 1869) & Pacific harvestfish & $110-222$ & $\mathrm{C}$ & POT \\
\hline Peprilus ovatus Horn, 1970 & Shining butterfish & $115-157$ & $\mathrm{R}$ & PS-PP \\
\hline Peprilus snyderi Gilbert \& Starks, 1904 & Salema butterfish & $49-179$ & $\mathrm{C}$ & PM-PP \\
\hline \multicolumn{5}{|l|}{ Order Pleuronectiformes } \\
\hline \multicolumn{5}{|l|}{ Suborder Pleuronectoidei } \\
\hline \multicolumn{5}{|l|}{ Family Paralichthyidae } \\
\hline Ancylopsetta dendritica Gilbert, 1890 & Three-spot flounder & $105-222$ & $\mathrm{R}$ & PM-PP \\
\hline Citharichthys platophrys Gilbert, 1891 & Small sandab & $33-124$ & $\mathrm{~F}$ & PM-PP \\
\hline
\end{tabular}


TABLE 1 (Continued) / CUADRO 1 (Continuación)

\begin{tabular}{|c|c|c|c|c|}
\hline Species & A & B & $\mathrm{C}$ & $\mathrm{D}$ \\
\hline Cyclopsetta panamensis (Steindachner, 1876) & God's flounder & $45-306$ & A & PC-PP \\
\hline Cyclopsetta querna (Jordan \& Bollman, 1890) & Toothed flounder & $56-385$ & $\mathrm{~F}$ & PC-PP \\
\hline Etropus crossotus Jordan \& Gilbert, 1882 & Fringed flounder & $89-170$ & $\mathrm{~F}$ & PS-PP \\
\hline Paralichthys woolmani Jordan \& Williams, 1897 & Speckled flounder & $225-290$ & $\mathrm{R}$ & PC-PP \\
\hline Syacium latifrons (Jordan \& Gilbert, 1882) & Beach flounder & $40-237$ & A & POT \\
\hline Syacium longidorsale Murakami \& Amaoka, 1992 & Longfin flounder & 96 & $\mathrm{R}$ & PM-PP \\
\hline Syacium ovale (Günther, 1864) & Oval flounder & $88-128$ & A & POT \\
\hline \multicolumn{5}{|l|}{ Family Bothidae } \\
\hline Bothus constellatus (Jordan, 1889) & Pacific eyed flounder & $35-303$ & A & PS-PP \\
\hline Engyophrys sanctilaurentii Jordan \& Bollman, 1890 & Speckled-tail flounder & $88-90$ & $\mathrm{R}$ & PC-PP \\
\hline Monolene asaedai Clark, 1936 & Asaedae flounder & $39-107$ & $\mathrm{~F}$ & PS-PP \\
\hline \multicolumn{5}{|l|}{ Family Cynoglossidae } \\
\hline Symphurus atramentatus Jordan \& Bollman, 1890 & Inkspot tonguefish & $130-135$ & $\mathrm{R}$ & PC-PP \\
\hline Symphurus atricaudus (Jordan \& Gilbert, 1880) & California tonguefish & $126-164$ & $\mathrm{C}$ & PO-PP \\
\hline Symphurus elongatus (Günther, 1868) & Elongate tonguefish & $72-266$ & $\mathrm{~F}$ & PM-PP \\
\hline Symphurus melanurus Clark, 1936 & Drab tonguefish & 135 & $\mathrm{R}$ & POT \\
\hline \multicolumn{5}{|l|}{ Family Achiridae } \\
\hline Achirus mazatlanus (Steindachner, 1869) & Mazatlan sole & $34-146$ & $\mathrm{C}$ & PS-PP \\
\hline Achirus scutum (Günther, 1862) & Network sole & $51-205$ & $\mathrm{~F}$ & PM \\
\hline Achirus zebrinus Clark, 1936 & Tehuantepec sole & $50-182$ & $\mathrm{~F}$ & PS-PP \\
\hline Trinectes fimbriatus (Günther, 1862) & Fringed sole & $57-75$ & $\mathrm{R}$ & PS-PP \\
\hline Trinectes fonsecensis (Günther, 1862) & Spottedfin sole & $58-190$ & $\mathrm{C}$ & PS-PP \\
\hline \multicolumn{5}{|l|}{ Order Tetraodontiformes } \\
\hline \multicolumn{5}{|l|}{ Suborder Balistoidei } \\
\hline \multicolumn{5}{|l|}{ Family Balistidae } \\
\hline Balistes polylepis Steindachner, 1876 & Finescale triggerfish & $45-350$ & $\mathrm{C}$ & PO-PP \\
\hline Pseudobalistes naufragium (Jordan \& Starks, 1895) & Stone triggerfish & $39-65$ & $\mathrm{C}$ & PS-PP \\
\hline \multicolumn{5}{|l|}{ Family Monacanthidae } \\
\hline Aluterus monoceros (Linnaeus, 1758) & Unicorn leatherjacket filefish & $280-441$ & $\mathrm{R}$ & $\mathrm{CT}$ \\
\hline \multicolumn{5}{|l|}{ Suborder Tetraodontoidei } \\
\hline \multicolumn{5}{|l|}{ Family Tetraodontidae } \\
\hline Sphoeroides annulatus (Jenyns, 1842) & Bullseye puffer & $30-264$ & $\mathrm{~F}$ & PM-PP \\
\hline Sphoeroides kendalli Meek \& Hildebrand, 1928 & Slick puffer & $65-87$ & $\mathrm{~F}$ & PS-PP \\
\hline Sphoeroides lobatus (Steindachner, 1870) & Longnose puffer & $46-210$ & $\mathrm{R}$ & PO-PP \\
\hline Sphoeroides sechurae Hildebrand, 1946 & Peruvian puffer & $44-169$ & $\mathrm{~F}$ & POT \\
\hline \multicolumn{5}{|l|}{ Family Diodontidae } \\
\hline Chilomycterus reticulatus (Linnaeus, 1758) & Spotfin burrfish & $60-190$ & $\mathrm{~F}$ & $\mathrm{CT}$ \\
\hline Diodon holocanthus Linnaeus, 1758 & Long-spine porcupinefish & $109-275$ & $\mathrm{R}$ & $\mathrm{CT}$ \\
\hline Diodon hystrix Linnaeus, 1758 & Spot-fin porcupinefish & $70-290$ & $\mathrm{C}$ & $\mathrm{CT}$ \\
\hline
\end{tabular}

A: Common Name in English; B: Size range (standard length, mm); C: Relative abundance; D: Province and biogeographical region (PO, Oregonian; PS, San Diego Province; PC, Cortés Province; PM, Mexican Province; PP, Panamic Province; POT, Tropical Eastern Pacific; CT, Circumtropical species; T, Trans-Pacific species). Species abundance: (A) abundant; (F) frequent; (C) common; (R) rare. Systematic arrangement according to Nelson (2006). Revision of species according to Eschmeyer (1998) and Froese \& Pauly (2013).

A: Nombre común en inglés, B: Intervalo de tallas (longitud estándar, mm), C: Abundancia relativa, D: Provincias y regiones biogeográficas (PO: Provincia Oregoniana, PS: Provincia de San Diego, PC: Provincia de Cortés, PM: Provincia Mexicana, PP: Provincia Panámica, POT: Pacífico Oriental Tropical, CT: Especies Circumtropicales, y T: Especies Transpacíficas). Especie abundante (A), especie frecuente (F), especie común (C) y especie rara (R). Clasificación sistemática según Nelson (2006). Revisión de especies según Eschmeyer (1998), Froese y Pauly (2013). 
TABLE 2

Comparison of previous studies regarding demersal fish lists in the continental shelf of the Gulf of Tehuantepec

CUADRO 2

Comparación de estudios anteriores con respecto a las listas de peces demersales en la plataforma continental del Golfo de Tehuantepec

\begin{tabular}{lccccccc}
\multicolumn{1}{c}{ Author } & Cruises & Year & $\begin{array}{c}\mathrm{N}^{\circ} \text { of } \\
\text { Trawls }\end{array}$ & $\begin{array}{c}\text { Depth } \\
\text { Range (m) }\end{array}$ & $\begin{array}{c}\mathrm{N}^{\circ} \text { of } \\
\text { Families }\end{array}$ & $\begin{array}{c}\mathrm{N}^{\circ} \text { of } \\
\text { Genus }\end{array}$ & $\begin{array}{c}\mathrm{N}^{\circ} \text { of } \\
\text { Species }\end{array}$ \\
Secretaria de Marina (1978) & 1 & 1977 & 11 & $27-60$ & 32 & 49 & 61 \\
Acal-Arias (1990) & 4 & 1987 & 161 & $15-200$ & 53 & 115 & 195 \\
Tapia-García (1998) & 5 & $1989-1990$ & 85 & $15-80$ & 56 & 123 & 173 \\
Present study & 15 & $2003-2005,2013$ & 344 & $15-64$ & 58 & 129 & 242 \\
\hline
\end{tabular}

fish species with respect to this study (Table 2). The Order Perciformes was the most diverse, with 20 families, 55 genera and 110 species, which is typical of the ichthyofaunal groups in the intertropical regions. The families best represented in terms of number of species were: Sciaenidae (29), Haemulidae (16), Carangidae (16), and Serranidae (14). The main genera were: Anchoa, Eucinostomus, Epinephelus and Urotrygon, each with six species, followed by Prionotus and Diplectrum, each with five species. Sizes ranged from $27 \mathrm{~mm}$ in the reddish scorpionfish (Scorpaena russula) to 681 $\mathrm{mm}$ in the Spotted-tail moray (Gymnothorax equatorialis). The species with the highest relative abundance were: Haemulopsis axillaris (Steindachner, 1869), Syacium ovale (Günther, 1864), Selene peruviana (Guichenot, 1866), Bothus constellatus (Jordan, 1889), Diapterus peruvianus (Cuvier and Valenciennes, 1830), Syacium latifrons (Jordan and Gilbert, 1882), Scorpaena russula (Jordan and Bollman, 1889), Eucinostomus currani (Zauranec, 1967), Haemulon scudderii (Gill, 1863), Prionotus stephanophrys (Lockington, 1881), and Larimus acclivis (Jordan and Bristol, 1898). $H$. axillaris, $S$. ovale, $S$. peruviana, D. peruvianus, L. acclivins and Stellifer erycimba were the most frequent species in the inner shelf $(<40$ $\mathrm{m}$ depth), whereas $P$. stephanophrys, S. russula, Porichthys analis and Synodus scituliceps were dominant in the outer shelf (40-60 m depth).

Individual fish size and weight showed a similar general pattern along the four years. Most of the fishes (40\%) presented a standard length range of 75 to $107 \mathrm{~mm}$ with a mean of $103 \pm 31.8$. Individual weight including $70 \%$ of the fishes varied between 1.25 to $32.5 \mathrm{~g}$ with a mean of $32.5 \pm 32.7$. Large fishes (> $50 \mathrm{~cm} \mathrm{SL}$ and $2000 \mathrm{~g}$ ) were also found in shrimp by-catch, although their abundance may be underestimated due to reduced catchability related to trawl speed and net selectivity. Most of these fishes were elasmobranch of commercial interest like Narcine vermiculatus, Gymnura marmorata and Rhinobathos glaucostigma, and teleosts like Epinephelus multiguttatus, Hyporthodus exsul, H. acanthisthius, Ophioscion typicus, Carangoides otrynter, Lutjanus guttatus, L. peru, Paralichthys woolmani, Cyclopsetta querna, Trichiurus lepturus, Centropomus robalito, Micropogonias altipinnis and Cynoscion phoxocephalus, which represented $25 \%$ of the biomass. Fish catches during day and night showed significant differences ( $\mathrm{t}$-test $=1.93, \mathrm{P}<0.05)$. Also catches recorded below $40 \mathrm{~m}$ depth presented a significant difference ( $\mathrm{t}$-test $=-5.67, \mathrm{P}<0.05)$ with those obtained deeper than $40 \mathrm{~m}$.

The community was composed mainly by species with subtropical and tropical affinities (Table 1). Subtropical and tropical fish represented $34.4 \%$ of the catch with a wide geographical distribution from the Cortés to the Panamic Province; $27.0 \%$ were eurythermic species from the San Diego to the Panamic Province, $13.3 \%$ were restricted to the Mexican and Panamic Provinces, and 9.3\% were species with a distribution in the Eastern Tropical Pacific (Fig. 2). The fish community 


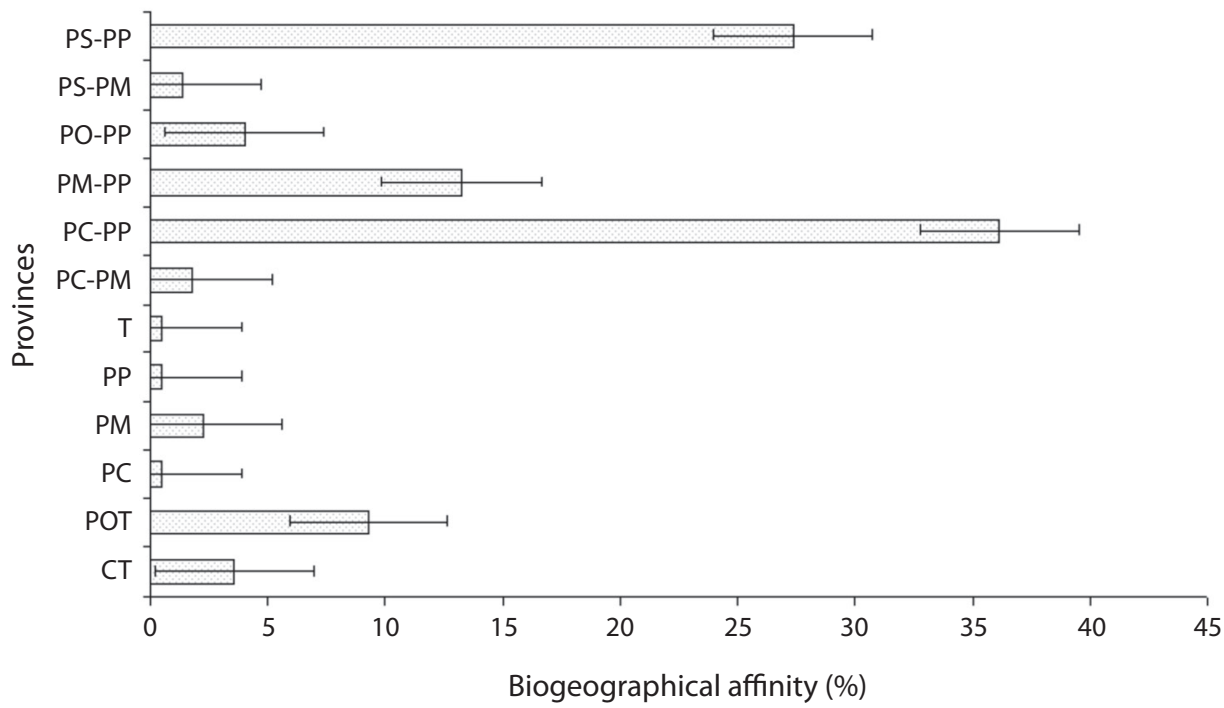

Fig. 2. Ichthyogeographic affinities (\%) in Provinces along the Eastern Pacific coast (PO, Oregonian; PS, San Diego; PC, Cortés; PM, Mexican; PP, Panamic; POT, Tropical Eastern Pacific; CT, Circumtropical species; T, Trans-Pacific species).

Fig. 2. Afinidad ictiogeográfica (\%) en las provincias a lo largo de la costa oeste del Pacífico (PO: Provincia Oregoniana, PS: Provincia de San Diego, PC: Provincia de Cortés, PM: Provincia Mexicana, PP: Provincia Panámica, POT: Pacífico Oriental Tropical, CT: Especies Circumtropicales, y T: Especies Transpacíficas).

was constituted by $32.8 \%$ of common species, whereas $28.6 \%$ are frequent species, 30.3 $\%$ are rare species and the remaining $8.3 \%$ were abundant.

New records for the area were: Notarius planiceps, Ophioscion typicus, Mugil setosus, Alphestes immaculatus, Chilara taylori, Decapterus muroadsi, Lepidopus fitchi and Stellifer chrysoleuca. The recorded distribution within the area was extended for Urotrygon reticulata, Anchoa helleri, Cathorops steindachneri, Bollmannia stigmatura and Sphoeroides kendalli.

In terms of abundance, the most important families were Haemulidae, Sciaenidae, Carangidae, Ariidae and Serranidae, which also contributed with the greatest number of species and abundance. These species are central to the understanding of the structure and function of the community of demersal fishes of the area. Its euryhaline capacity explains its abundance in front of the lagoons of the Huave, Mar Muerto, La Joya-Buenavista, Carretas-Pereyra and Chantuto-Panzacola systems.
Of the 242 species recorded, 70 (30\%) made incursions into the coastal lagoons for various purposes such as spawning and feeding. Notable for their abundance were those in the Huave (Oaxaca) and Chantuto Panzacola (Chiapas) systems: Lile stolifera, L. gracilis, Diapterus peruvianus, Micropogonias altipinnis, Achirus zebrinus, A. scutum, Anchoa nasus, A. mundeola, Centropomus robalito, Cathorops fuerthii, Eucinostomus currani and Cyclopsetta panamensis.

Three species (1.3\%) were typically freshwater species: Poecilia butleri, Poecilopsis fasciata and Gobiomorus maculatus. They were caught near the outlet of the Mar Muerto lagoon at $16-18 \mathrm{~m}$ depth, possibly due to the influence of a plume of brackish water intruding the continental shelf.

The total number of species registered in 2003 (131) was higher than the one found in 2013 (116), whereas species biogeographical affinities did not present a significant difference $\left(\mathrm{F}_{12,9}=4.41\right.$, d.f. $\left.=21.0, \mathrm{P}>0.447\right)$ among 2003 and 2013. 


\section{DISCUSSION}

The species richness of demersal fishes recorded as by-catch from the shrimp fishery in the Gulf of Tehuantepec, with 242 species, is typical of the Eastern Pacific (Robertson \& Cramer, 2009). Only 34 species (15\%) were frequent or abundant while the others were common and rare. Some of the rare species are epipelagic species (Opisthonema libertate, Scomberomorus sierra, Decapterus macarellus and Cypselurus callopterus), which probably may have been caught while the net was being recovered.

Fish by-catch individual size and weight were consistently small, with $92 \%$ under 50 g weight and $20 \mathrm{~cm} \mathrm{SL}$, respectively. A similar composition has been previously reported for the Gulf of California (Pérez Mellado \& Findley, 1985). These authors pointed out that about $1 \%$ were fishes weighting more than $100 \mathrm{~g}$ whereas in this study the percentage was remarkably higher (3.4\%). These fishes, usually higher than $350 \mathrm{~g}$, were frequent in by-catch and are mainly used for human consumption.

On previously compiled lists of species for the demersal fishery on the continental shelf in the Gulf of Tehuantepec, recorded 32 families, 49 genera and 61 species (Secretaria de Marina, 1978). Acal and Arias (1990) registered 292 species, but that list contained 97 inconsistencies; 50 species were not identified but simply assigned to genus, 24 others were only assigned to family, six constituted synonyms within the list, and others were clearly out of context since they were species recorded for the Atlantic Ocean; the record has accordingly been adjusted.

Of the species in previous lists from the Gulf of Tehuantepec, 167 (74 \%) were also found in the present study but 99 were not. Compiling these studies we obtained a total of 331 species, which supports the fact that recorded fish species in the Mexican South Pacific are higher than those for the Western shelf of Baja California Sur, the Gulf of California, the Jalisco and Colima shelf, and the shelves of Nayarit, Michoacán and Guerrero.
This number may be greater if species that belong to the rocky, pelagic and slope zones, and to the lagoons and estuaries, are considered.

From a biogeographical point of view, the assignment of this ichthyofauna to the classical faunistic provinces (Cortés, Panamic and Mexican) presents difficulties, particularly because it is constituted by species with tropical affinities that overlap with others with subtropical or temperate affinities. In addition, it must be considered that the conjuction of provinces (Cortés-Panamic) corresponds to 12 zoogeographic divisions. This marks a distinct change in association, frequency and diversity of species from South to North, reflecting the great variety of habitats.

In the Northern area, the species were temperate ones: Bellator xenisma, Diplectrum pacificum, Epinephelus analogus, Sphoeroides lobatus, Synodus evermanni, G. marmorata, Ophidion galeoides, Opisthonema medirastre and Merluccius productus. To the South, the predominant species are typical of the Tropical Eastern Pacific, such as Bellator loxias, Porichthys analis, Ancylopsetta dendritica, Aluterus monoceros, Trinectes fimbriatus, Diplectrum macropoma, Urotrygon munda and Paralonchurus rathbuni. Horn et al. (2006) and Rodríguez-Romero, HernándezVázquez and López-Martínez (2009) noted similar tendencies.

The high diversity recorded in the Gulf of Tehuantepec, while demonstrating the importance and uniqueness of the area, reflects the concurrence of a series of environmental factors that interact, characterizing the region as a dynamic frontier or transition ecosystem with a broad thermic regime and a variety of habitats (Díaz-Ruiz, Cano-Quiroga, AguirreLeón, \& Ortega-Bernal, 2004; Tapia-García, García-Abad, Carranza-Edwards \& VázquezGutiérrez, 2007; Velázquez-Velázquez, VegaCendejas \& Navarro-Alberto, 2008). This uniqueness is supported not only by its geographic position with tropical and temperate characteristics, but also by: 1) the contribution of river runoff, 2) the presence of extensive coastal lagoon systems with high productivity, 
3) the prevailing winds and seasonal rainfall from land to the sea, and 4) a wide continental shelf influenced by a complex oceanic current system (the sub-surface equatorial, the North-equatorial countercurrent, the California current, and the South-equatorial or coastal current of Costa Rica that flows northwards and favors the presence of important upwellings) (Galván-Magaña et al., 2000; López-Martínez, Herrera-Valdivia, Rodríguez-Romero, \& Hernández-Vázquez, 2010; Mora \& Robertson, 2005; Rodríguez-Romero et al. 1998; Rodríguez-Romero, Palacios-Salgado, LópezMartínez, Hernández-Vázquez, \& Ponce-Díaz, 2008; Zapata \& Robertson, 2007).

In addition, the species of the Tropical Eastern Pacific receive strong immigration on its coasts, particularly during events such as ENSO (El Niño - Southern Oscillation), which reach the East Pacific coasts when it is of great magnitude and intensity (Lea \& Rosenblatt, 2000). Their periodicity and magnitude contribute significantly to dispersion, since most fishes have life cycles with pelagic larval stages (Auster, 1988; Caddy \& Sharp, 1986; Galzin \& Legendre, 1987; Grossman, Freeman, Moyle, \& Whitaker, 1985), which affect the distribution patterns since long time. It is considered that changes in abundance and composition of the fish community frequently occur as a result of migratory movements between neighbouring geographic areas driven by climatic and oceanographic processes of medium or longterm duration (Safran, 1990), as well as other factors such as changes in the rates of hatching and mortality, owing to abiotic environmental factors (temperature, oxygen) or biotic factors (predation, competition, food availability).

The results show the uniqueness and importance of the region in relation to the fish species richness of great value for industrial and artisanal fisheries. In this context, it would be important to include, in the actual local policies, rules that could regulate the technical aspects of the fishing methods used in the Gulf of Tehuantepec in order to preserve this ecosystem richness. Consequently, scientific research appropriate to the particular needs of this area of the Tropical Eastern Pacific should increase, so long as the information is scarce and temporarily discontinuous.

\section{ACKNOWLEDGMENTS}

To the University of Barcelona for a collaborative grant, in particular to the Department of Ecology for the help in its computer section, library and laboratories; without these facilities it would have been difficult to proceed with the doctoral study of the first author. Thanks to the Institute of Marine Sciences of Barcelona, particularly the Department of Renewable Marine Resources of CSIC for granting the support and infrastructure necessary for this study. To the Regional Centre of Fisheries Research, Salina Cruz, of the Mexican National Institute of Fisheries and its personnel, for the opportunity to participate in the fishing expeditions in 2003 and 2005, and for the help in the laboratory and hospitality in their premises. Thanks to the Postgraduate Department of the Institute of Marine Sciences and Limnology, UNAM, for the assistance during a postdoctoral residency and for the translation of the manuscript. This study was partially supported by the Dirección General de Asuntos del Personal Académico, Universidad Nacional Autónoma de México (PAPIIT), Grant IN211214-2.

\section{RESUMEN}

En la pesquería del camarón del Golfo de Tehuantepec un gran número de especies de peces se capturan como fauna de acompañamiento y son descartadas. La composición, distribución y afinidades biogeográficas de la ictiofauna acompañante del camarón fue analizada mediante 15 cruceros desarrollados en la plataforma continental entre 15-64 m de profundidad durante 2003, 2004, 2005 y 2013. La ictiofauna descartada estuvo representada por 58 familias, 129 géneros y 242 especies. Las familias, Haemulidae, Sciaenidae, Paralichthyidae, Gerreidae y Carangidae aportaron más del $70 \%$ de la captura. Haemulopsis axillaris, Syacium ovale, Selene peruviana, Diapterus peruvianus, Larimus acclivins y Stellifer erycimba fueron las especies más frecuentes en profundidades menores de $40 \mathrm{~m}$ (plataforma interna), mientras que Prionotus stephanophrys, Scorpaena russula, Porichthys analis y Synodus scituliceps, fueron dominantes entre $40-60 \mathrm{~m}$ de profundidad 
(plataforma externa). El análisis de las afinidades biogeográficas mostró que el $36.1 \%$ de las especies son de amplia distribución desde la provincia de San Diego a la Panámica, mientras que el $13.2 \%$, presentó una distribución restringida entre la provincia Mexicana y la Panámica. La composición de la ictiofauna estuvo marcadamente influenciada por las condiciones ambientales locales y sus variaciones estacionales.

Palabras clave: biogeografia, ictiofauna, camarón, pesca, fauna de acompañamiento, Golfo de Tehuantepec.

\section{REFERENCES}

Acal, E. D. \& Arias, A. (1990). Evaluación de los recursos demerso-pelágicos vulnerables a redes de arrastre de fondo en el sur del Pacífico de México. Ciencias Marinas, 16, 93-129.

Aguilar-Palomino, B., Mariscal, R. J., González, S. G., \& Rodríguez, I. L. E. (1996). Lista sistemática de la ictiofauna demersal de fondos blandos de la plataforma continental de Jalisco y Colima México, durante mayo y junio de 1995. Ciencias Marinas, $22,469-481$.

Allsop, W. H. L. (1985). La fauna acompañante del camarón: perspectivas y manejo. En A. Yánez-Arancibia (Ed.). Recursos pesqueros potenciales de México: La pesca acompañante del camarón. Programa Universitario de Alimentos (pp. 635-643). México D.F: Instituto de Ciencias del Mar y Limnología, Instituto Nacional de la Pesca, UNAM.

Amezcua-Linares, F. (1996). Peces demersales de la Plataforma Continental de México. México: UNAM/ ICMYL/CONABIO.

Andrew, N. L., \& Pepperell, J. G. (1992). The by-catch of shrimp trawl fisheries. Oceanography and Marine Biology: An Annual Review, 30, 527-565.

Auster, P. J. (1988). A review of present state of understanding of marine fish communities. Journal of Northwest Atlantic Fisheries Science, 8, 67-75.

Bianchi, G. (1991). Demersal assemblages of the continental shelf and slope edge between the Gulf of Tehuantepec (México) and Gulf of Papagayo (Costa Rica). Marine Ecology Progress Series, 73, 121-140.

Boschi, E. E. (2000). Species of decapod crustaceans and their distribution in the American marine zoogeographic provinces. Revista de Investigación y Desarrollo Pesquero, 13, 7-136.

Briggs, J. C. (1974). Marine Zoogeography. NewYork: McGraw-Hill.

Briggs, J. C. (1995). Global biogeography. Amsterdam, Holland: Elsevier.
Bussing, W., \& López, M. (1993). Peces demersales y pelágicos costeros del Pacífico de Centro América Meridional. Revista de Biología Tropical, Publicación Especial, 1, 1-164.

Caddy, J. F., \& Sharp, G. D. (1986). An ecological framework for marine fishery investigations. FAO Fisheries Technical Paper, 283, 152.

Castro-Aguirre, J. L. (1978). Catálogo sistemático de los peces marinos que penetran a las aguas continentales de México, con aspectos zoogeográficos y ecológicos. México: Instituto Nacional de la Pesca, Depto. Pesca.

Castro-Aguirre, J. L. \& Espinosa Pérez, H. (1996). Listados faunisticos de México. VII. Catálogo sistemático de las rayas y especies afines de México (Chondrichthyes: Elasmobranchii: Rajiformes: Batoideiomorpha). México, D.F: Instituto de Biología, UNAM.

Cervantes-Hernández, P., Gallardo-Berumen, M. I., Ramos-Cruz, S., Gómez-Ponce, M. A., \& Gracia, A. (2008). Análisis de las temporadas de veda en la explotación marina de camarones del Golfo de Tehuantepec, México. Revista de Biología Marina y Oceanografia, 43, 285-294.

Díaz-Ruiz, S., Cano-Quiroga, E., Aguirre-León, A., \& Ortega-Bernal, R. (2004). Diversidad, abundancia y conjuntos ictiofaunísticos del sistema lagunar-estuarino Chantuto-Panzacola, Chiapas, México. Revista de Biología Tropical, 52, 187-199.

Eschmeyer, W. N. (1998). Catalog of fishes. Special Publication, 1. San Francisco: California Academy of Sciences.

Espinosa-Pérez, H. J., Castro-Aguirre, L., \& HuidobroCampos, L. (2004). Listados faunísticos de México. IX. Catálogo sistemático de tiburones (Elasmobranchii: Selachimorpha). México, D.F., México: Instituto de Biología UNAM.

Fischer, W., Krupp, F., Schneider, W., Sommer, C., Carpenter, K. E., \& Niem, V. H. (1995). Guía FAO para la identificación de especies para los fines de la pesca Pacifico centro-oriental (Volumen III. Vertebrados, Parte 2). Roma: FAO.

Froese, R., \& Pauly, D. (2013). FishBase. Retrieved from http://www.fishbase.org.

Galván-Magaña, F., Gutiérrez-Sánchez, F. J., Abitia-Cárdenas, L. A., \& Rodríguez-Romero, J. (2000). The distribution and affinities of the shore fishes of Baja California Sur lagoons. In M. Munawar, S. G. Lawrence, I. F. Munawar, \& D. F. Malley (Eds.). Aquatic ecosystems of Mexico (pp. 383-398). Leiden: Ecovision world monograph series. Backhuys Publishers.

Galzin, R., \& Legendre, P. (1987). The fish communities of a coral reef transect. Pacific Science, 41, 158-165. 
Ginsburg, I. (1958). Flounders of the genus Paralichthys and related genera in American waters. U.S. Fisheries and Wildlife Service, Fishery Bulletin, 52, 267-351.

Griffiths, S., Larson, H., \& Courtney, T. (2004). Trawl bycatch species in National Oceans Office, Description of key species groups in the northern planning area. Hobart, Australia: National Oceans Office.

Grossman, G. D., Freeman, M. C., Moyle, P. B., \& Whitaker Jr., J. O. (1985). Stochasticity and assemblage organization in an Indiana stream fish assemblage. American Naturalist, 126, 275-285.

Hastings, P. A. (2000). Biogeography of the tropical eastern Pacific: distribution and phylogeny of chaenopsid fishes. Zooogical. Journal of Linnean Society, 128, 319-335.

Hendrickson, H. M., \& Griffin, W. L. (1993). An analysis of management policies for reducing shrimp by-catch in the Gulf of Mexico. North American Journal of Fisheries Management, 13, 686-697.

Horn, M. H., Allen, L. G., \& Lea, R. N. (2006). Biogeography. In L. G. Allen, D. J. Pondella, \& M. H. Horn (Eds.). The ecology of marine fishes, California and adjacent waters (pp. 3-25). California, U.S.A.: University of California Press, Berkeley.

Lea, R. N., \& Rosenblat, R. H. (2000). Observations on fishes associated with the 1997-98 EI Niño off California. CalCOFI Report, 41, 117-129.

López-Martínez, J., Herrera-Valdivia, E., RodríguezRomero, J., \& Hernández-Vázquez, S. (2010). Composición taxonómica de peces integrantes de la fauna de acompañamiento de la pesca industrial de camarón del Golfo de California, México. Revista de Biología Tropical, 58, 925-942.

Love, M. S., Mecklenburg, C. W., Mecklenburg, T. A., \& Thorsteinson, L. K. (2005). Resource Inventory of Marine and Estuarine Fishes of the West Coast and Alaska: A Checklist of North Pacific and Arctic Ocean Species from Baja California to the AlaskaYukon Border. Washington: U. S. Department of the Interior, U. S. Geological Survey, Biological Resources Division, Seattle, Washington.

McPhail, J. D. (1958). Key to the croakers (Sciaenidae) of the eastern Pacific. University of British Columbia. Institute of Fisheries, Museum Contributions, 2, 1-20.

Madrid-Vera, J., Ruiz, A. L., \& Rosado, B. I. (1998). Peces de la plataforma continental de Michoacán y sus relaciones en el Pacífico mexicano. Revista de Biología Tropical, 46, 267-276.

Moncayo-Estrada, R., Castro-Aguirre, J. L., \& De La CruzAgüero, J. (2006). Lista sistemática de la ictiofauna de Bahía de Banderas, México. Revista Mexicana de Biodiversidad, 77, 67-80.
Mora, C., \& Robertson, D. R. (2005). Causes of latitudinal gradients in species richness: a test with the endemic shorefishes of the Tropical Eastern Pacific. Ecology, $86,1771-1782$.

Nelson, J. S. (2006). Fishes of the world. New Jersey, USA: John Wiley \& Sons.

Nelson, J. S., Crossman, E. J., Espinosa-Pérez, H., Findley, L. T., Gilbert, C. R., Lea, R. N., \& Williams, J. D. (2004). Common and scientific names of fishes from the United States, Canada, and Mexico. American Fisheries Society. Special Publication, 29, Maryland, EUA.

Norman, J. R. (1934). A systematic monograph of the flatfishes (Heterosomata) Vol. I. Psettodidae, Bothidae, Pleuronectidae. London: British Museum Natural History.

Pérez-Mellado, J., \& Findley, L. T. (1985). Evaluación de la ictiofauna acompañante del camarón capturado en la costa de Sonora y norte de Sinaloa, México. En A. Yánez-Arancibia (Ed.), Recursos pesqueros potenciales de México: La pesca acompañante del camarón (pp. 201-254). México: PUA, ICMYL, UNAM/INP.

Reyna Cabrera, I. E., \& Ramos-Cruz, S. (1998). La pesquería de camarón de alta mar. En M. Tapia-García (Ed.), El Golfo de Tehuantepec: el ecosistema y sus recursos (pp. 163-178). México: Universidad Autónoma Metropolitana-Iztapalapa.

Robertson, D. R., \& Allen, G. R. (2002). Shore fishes of the Tropical Eastern Pacific: an Information System. CD-ROM. Panamá: Smithsonian Tropical Research Institute, Balboa, Panamá.

Robertson, D. R., \& Cramer, K. L. (2009). Shore fishes and biogeographic subdivisions of the Tropical Eastern Pacific. Marine Ecology Progress Series, 380, 1-17.

Robertson, D. R., Grove, J. S., \& McCoster, J. S. (2004). Tropical trans-Pacific shore fishes. Pacific Science, 58, 507-565.

Rodríguez-Romero, J., Abitia, C. L., Galván, M. F., Gutiérrez, S., Aguilar, P. B., \& Arvizú, M. J. (1998). Ecology of fish communities from the soft bottoms of Bahía Concepción, México. Archive of Fishery Marine Research, 46, 61-76.

Rodríguez-Romero, J., Palacios-Salgado, D. S., LópezMartínez, J., Hernández-Vázquez, S., \& Ponce-Díaz, G. (2008). Composición y zoogeografía de los peces demersales de la costa occidental de Baja California Sur, México. Revista de Biología Tropical, 56, 1765-1783.

Rodríguez-Romero, J., Hernández-Vázquez, S., \& LópezMartínez, J. (2009). Desarrollo potencial de peces desaprovechados. Ciencia y Desarrollo, 35, 45-51. 
Rosemblatt, R. H., \& Johnson, G. D. (1974). Two new species of sea basses of the genus Diplectrum with a key to the Pacific species. California Fish and Game, 60, 178-191.

Safran, P. (1990). Spatio-temporal variability in the structure of a nectobenthic fish nursery: A descriptive study. Oceanologica Acta, 13, 97-106.

Sarmiento-Náfate, S., Gil-López, H. A., \& Arroyo, D. (2007). Shrimp by-catch reduction using a short funnel net, in the Gulf of Tehuantepec, South Pacific, Mexico. Revista Biologia Tropical, 55, 889-897.

Secretaria de Marina. (1978). Estudio oceanográfico del Golfo de Tehuantepec. Tomos I-III. (Biología, Física, y Química; Geología). México: Secretaria de Marina. Dirección General de Oceanografía. Talleres de la Nación, México.

Siqueiros-Beltrones, D. A., \& De la Cruz Agüero, J. (2004). Examen filosófico de las listas sistemáticas como informes científicos y porqué deben ser publicados. Oceánides, 19, 1-9.

Sokal, R. R., \& Rohlf, F. J., (1995). Biometry. New York, USA: W.H. Freeman and Co.

Stobutzki, I. C., Miller, M. J., Jones, P., \& Salini, J. P. (2001). Bycatch diversity and variation in a tropical Australian peneaeid fishery; the implications for monitoring. Fisheries Research, 53, 283-301.

Tapia-García, M., García-Abad, M. C., González-Medina, G., Macuitl-Montes, M. C., \& Cerdenares-Ladrón de Guevara, G. (1994). Composición, distribución y abundancia de la comunidad de peces demersales del Golfo de Tehuantepec. Tropical Ecology, 35, 229-252.
Tapia-García, M. (1998). Evaluación ecológica de la fauna demersal. In M. Tapia-García (Ed.), El Golfo de Tehuantepec: el ecosistema y sus recursos (pp. 129-148). México, D. F: Universidad Autónoma Metropolitana-Iztapalapa.

Tapia-García, M., \& García-Abad, M. C. (1998). Los peces acompañantes del camarón y su potencial como recurso en las costas de Oaxaca y Chiapas. In M. Tapia-García (Ed.), El Golfo de Tehuantepec: el ecosistema y sus recursos (pp. 179-196). México, D.F: Universidad Autónoma Metropolitana-Iztapalapa.

Tapia-García, M., García-Abad, A., Carranza-Edwards, A., \& Vázquez-Gutiérrez, F. (2007). Environmental characterization of the continental shelf of the gulf of Tehuantepec, Mexico. Geofisica Internacional, $7,249-260$

Velázquez-Velázquez, E., Vega-Cendejas, M. E., \& Navarro-Alberto, J. (2008). Spatial and temporal variation of fish assemblages in a coastal lagoon of the Biosphere Reserve La Encrucijada, Chiapas, Mexico. Revista de Biología Tropical, 56, 557-574.

Walker, B. W. (1960). The distribution and affinities of the marine fish fauna of the Gulf of California. Systematic Zoology, 9, 123-133.

Walter, H. J., \& Rosemblatt, R. H. (1988). Pacific toadfishes of the genus Porichthys (Batrachoididae) with description of three new species. Copeia, 4, 887-904.

Zapata, F. A., \& Robertson, D. R. (2007). How many species of shore fishes are there in the Tropical Eastern Pacific? Journal of Biogeography, 34, 38-51.

Zar, J. H. (1999). Biostatistical analysis. New York, USA: Prentice Hall. 